\title{
A Study of the Signal Information by ULV-SEM in Ultra Low Landing Voltage
}

Shuichi Takeuchi*, Atsushi Miyaki*, Atsushi Muto*, Satoshi Okada*, Michio Hatano** and Sukehiro Ito*

*Hitachi High-Technologies Corp., 11-1 Ishikawa-cho, Hitachinaka, Ibaraki 312-0057, Japan **Hitachi, Ltd., Central Research Laboratory 1-280 Higashi-koigakubo, Kokubunji, Tokyo, 1858601 Japan

Ultra low voltage scanning electron microscopy (ULV-SEM) effectively works on the enhancement of electron beams generated from the specimen surface due to the reduction of the penetration depth of incident electron beam. ULV-SEM is widely used to various fields such as environmentally friendly materials, semiconductors and nano-materials, where the surface structures of the order of a few nanometers or less is important for evaluation. On the other hand, a study of signal information in ULV condition is important because the signal behavior at ULV doesn't follow the theory of conventionally used voltages over $1 \mathrm{kV}[1]$.

To study the physical characteristics of detection signal under ULV condition, we used an SU8000 FE-SEM to obtain the ULV-SEM images and analyzed by the electron simulation technique.

The SU8000 FE-SEM is offering the low energy and high energy filtering capability even at ultra low voltage condition as shown in Fig.1[2]. The top detector detects low energy electron signals while the upper detector detects high energy electron signals. The electron simulation provides the angle and energy information detected to the top and the upper detector respectively.

Fig. 2 shows the upper and the top detector imaging of the anode parts of Li ion battery at the landing voltage of $100 \mathrm{~V}$. It is composed by carbon graphite and binder which includes fluoride compounds. In the upper detector image (a), topographic images of the graphite surface and the stacking of plateshaped graphite are clearly observed. In the image by the top detector (b), some further structures of graphite surface were visualized with network distribution of dark dots.

The simulation results suited the difference of image quality obtained with each detector and emphasized that these images could show the characteristic difference of signals. We can expect that electrical potential information is rich in top detector image so that dark dots might be the fluoride components visualized by the potential contrast.

References

[1] Ilona Müllerová, Scanning 23, 379, (2001).

[2] A Miyaki et al., Microsc. Microanal. 15(Suppl 2), 2008, 662CD. 


\section{Top detector}

(Low energy electrons)

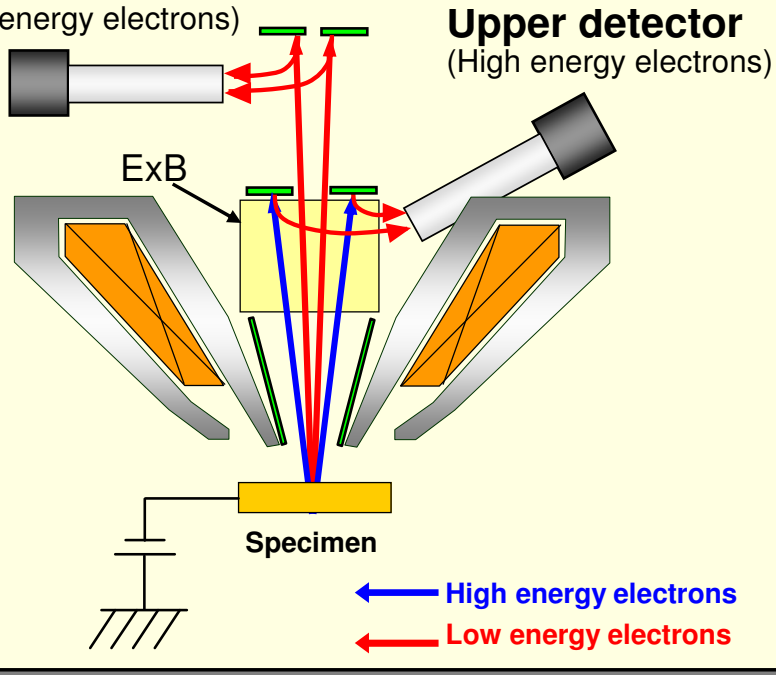

Fig.1 Signal detection system in SU8000 FE-SEM.
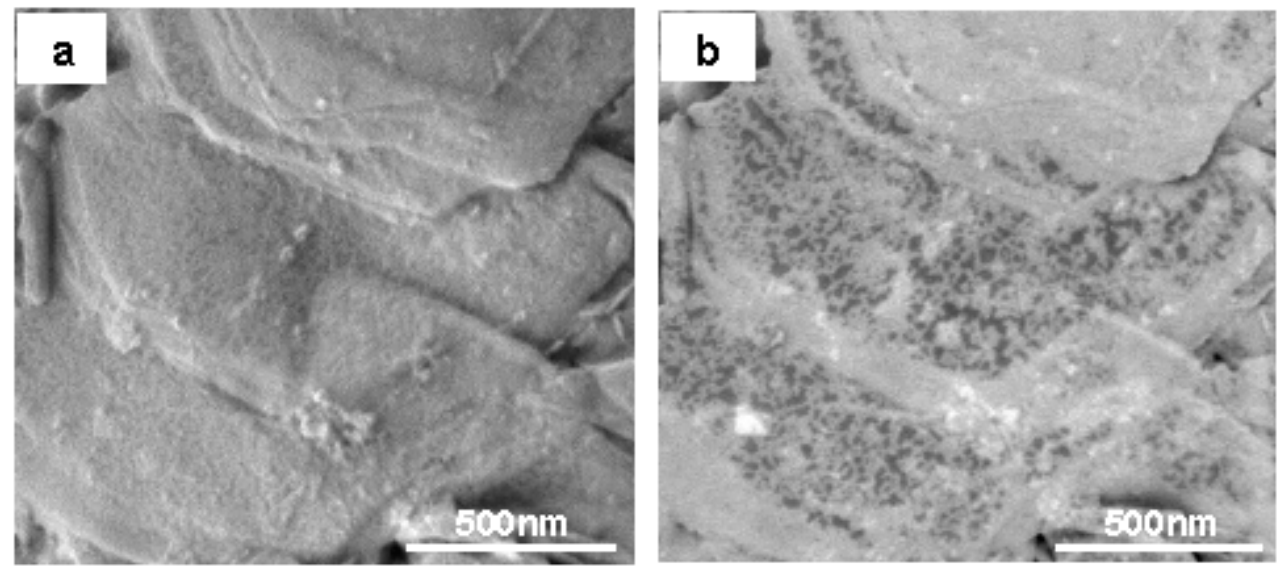

Fig. 2 SEM image of the anode part of Li ion battery at the landing voltage of $100 \mathrm{~V}$.

(a)Upper detector image, (b)Top detector image. 